\title{
Security Analysis and Implementation of an Improved Cch2 Proxy Multi-Signature Scheme
}

\author{
Raman Kumar \\ Department of Computer Science and Engineering, D A V Institute of Engineering and Technology, Jalandhar, Punjab, India. \\ er.ramankumar@aol.in
}

\begin{abstract}
Many of the signature schemes are proposed in which the $\mathrm{t}$ out of $\mathrm{n}$ threshold schemes are deployed; but they still lack the property of security. In this paper, we have discussed implementation of improved $\mathrm{CCH} 1$ and improved $\mathrm{CCH} 2$ proxy multi-signature scheme based on elliptic curve cryptosystem. We have represented time complexity, space complexity and computational overhead of improved $\mathrm{CCH} 1$ and $\mathrm{CCH} 2$ proxy multi-signature schemes. We have presented cryptanalysis of improved $\mathrm{CCH} 2$ proxy multi-signature scheme and showed that improved $\mathrm{CCH} 2$ scheme is suffered from various attacks i.e. forgery attack and framing attack.
\end{abstract}

Index Terms - Proxy Signature; Enforceability; Secret Sharing.

\section{INTRODUCTION}

Elliptic curve cryptosystem was introduced by Koblitz [1] and Miller [2] in 1985. The attractive public key cryptosystem is ECC because ECC has shorter key size and faster computing speed. The public key cryptosystem's security is based on the relative complexity of the mathematical problem. For example, the security of RSA depends on integer factorizing problem and the security of DSA depends on discrete logarithm problem [10]. ECC is developed by integer points over elliptic curves in finite fields. The security of ECC is based on the difficulty of solving the elliptic curve discrete logarithm problem (ECDLP). When one person needs to delegate his/her signing capability to other party or person, the proxy signature is very useful tool. In 1996, Mambo et al.'s [3] introduced first proxy signature scheme. Proxy signature is a signature scheme which allows one party called original signer to delegate his/her signing capability to another party called Proxy signer and then on behalf of the original signer, proxy signer can create signature on messages and after signature creation, proxy signer sends these signatures to the verifier and then verifier verify these signatures. Yi et al.'s [6] presented proxy multi-signature scheme which enables one proxy signer to create signature on behalf of group of original signers.

\section{Various security definitions:}

A proxy signature should have security properties [4] and they are:

1. Strong Unforgeability: Only designated proxy signer can create proxy signatures. Original signer or any other party cannot create proxy signatures.

2. Verifiability: A verifier can be convinced of the original signer's agreement on the signed message from the proxy signature.

3. Strong Identifiability: From the proxy signature, anyone can determine the identity of corresponding proxy signer.

4. Strong Undenability: once valid proxy signature is created by proxy signer for an original singer, he/she cannot repudiate signature creation.

5. Distinguishability: Proxy signatures that are created by proxy signer are distinguishable from ordinary signatures that are created by original signer.

\section{Various attacks:}

- Public key substitution attack: By updating his/her own public key, an attacker can forge a valid proxy multi-signature [7].

- Original signer's forgery attack: Without agreement of proxy signer, original signers can create proxy multi-signature. Verifier will be sure that any proxy multi-signature created by using forged signing key are created by agreement of all original signers and proxy signer [15]. Under the name of proxy signer, original signer can forge valid proxy multisignature.

- Transferring attack: In this attack, proxy signer's standard schnorr signature can be converted into proxy signature in which signer is regarded as proxy signer by the verifier and vice versa [13].

- Framing attack: In this attack, any user P can be framed by malicious users $A_{1}, A_{2} \ldots A_{n}$. User $P$ does not receive any delegation from the users $A_{1}, A_{2} \ldots A_{n}$, but the malicious users $A_{1}, A_{2} \ldots A_{n}$ can forge a proxy multi-signature for message by user $P$ on behalf of users $A_{1}, A_{2} \ldots A_{n}[16]$. 
Mambo et al.'s [3] introduced different types of delegations i.e. full delegation, partial delegation and delegation by warrant. In full delegation, the original signer gives his/her own secret key to proxy signer, so that proxy signer can create same signature as original signer creates. In partial delegation, a proxy signer has proxy private key, which is different from original signer's private key and proxy signer can sign range of messages in partial delegation because delegation period is not specified. In delegation by warrant, warrant is added that specifies what kinds of messages are delegated, the delegation period, IDs of original signers and proxy signer etc. According to protection of proxy signer, partial delegation is further divided as proxyunprotected and proxy-protected. In proxy unprotected, the original signer gives proxy signing key to the proxy signer and proxy signer create signature by using that key. So, in proxy unprotected proxy signature the original signer knows the proxy signing key and he/she can also create the same proxy signatures. In proxy protected, the proxy signer compute proxy signing key from his/her owns private key and the key given by original signer and then generates proxy signature. So in proxy protected proxy signature any third party including the original signer cannot create the same proxy signatures. In partial delegation, the signing capability of the proxy signer can be revoked by the original signer by two ways i.e. (1) Prepare revocation list and make it publicly seen. (2) The public key of original signer can be changed and accordingly all proxies of sincere proxy signers are updated. M. Mambo, K. Usuda, and E. Okamoto [4] have introduced proxy protected proxy signature scheme based on discrete logarithm problem. S. Kim, S. Park, and D. Won [5] have introduced two new types of proxy signature schemes i.e. proxy signature for partial delegation with warrant and proxy signature for threshold delegation. Partial delegation with warrant combines the benefits of partial delegation and delegation with warrant. H. M. Sun [7] analyzes Yi et al.'s [6] proxy multi-signature schemes and show that these schemes are suffered from public key substitution attack and direct forgery attack. They introduced a new proxy protected and proxy unprotected proxy multi-signature schemes which do not suffered from these attacks. B. Lee, H. Kim, and K. Kim [8] develop a strong non-designated proxy signature scheme and apply it to multi-proxy signature in which multiple original signers can delegate his signing capabilities to undetermined proxy signers. T. S. Chen, T. P Liu, and Y. F Chung [9] introduced a proxy protected proxy signature scheme based on elliptic curve discrete logarithm problem. On the basis of time complexity, they compare H. M. Sun [7] and the proposed scheme. T.S. Chen, Y.F. Chung and G.S. Huang [10] introduced an improved scheme in which the exponential operations are replaced by elliptic curve multiplicative ones. ECC with lower computational overhead and a smaller key size can achieve a level of security equal to that of the RSA or DSA. This proposed scheme is called CCH1 scheme. Without loss of security, the time complexity of proposed scheme is reduced and performance is enhanced. T.S. Chen, Y.F. Chung, G.S. Huang [11] introduced a traceable proxy multi-signature scheme. This proxy signature scheme is independent of number of original signers so, number of operations required for verification decreases. This proposed scheme is called $\mathrm{CCH} 2$ scheme. On the basis of time complexity they compare the sun's [7] and proposed proxy multi-signature scheme. M.S. Hwang, S.F. Tzeng and C.S. Tsai [12] introduced a generalized version of the $(\mathrm{t} 1 / \mathrm{n} 1-\mathrm{t} 2 / \mathrm{n} 2)$ proxy signature scheme based on elliptic curve discrete logarithm problem. G. L. Wang, F. Bao, J. Y. Zhou, and R. H. Deng [13] analyze the security of some proxy signature schemes i.e. M. Mambo et al.'s [4] and B. Lee et al.'s [8] and show that all these schemes are suffered from various attacks. S. Wang, G. Wang, F. Bao and J. Wang [14] shows that chen et al.'s [9] is vulnerable to an original signer forgery attack and introduced an improved scheme which is secure against the proposed attack. J.H. Park, B.G. Kang, S. Park [15] shows that proxy multisignature schemes proposed by Chen et al.'s $[10,11]$ are vulnerable to forgery attack by one or all original signers. M.H. Chang, I.T. Chen and M.T. Chen [17] presented a proxy protected signature scheme based on ECDSA which satisfies security properties. Fengying Li, Qingshui Xue [18] proposed improved CCH1 and improved $\mathrm{CCH} 2$ schemes that are not suffered from forgery attack. Tutanescu, C. Anton, L. Ionescu and D. Caragata [19] examines that ECC is an attractive public key cryptosystem than RSA or DSA for mobile devices, which have limited memory, processing capability and network connectivity. ECC can be implemented with less hardware, because of shorter key length. They have defined the application of ECC i.e. internet, smart cards, PDAs and PCs.

\section{TIME, SPACE AND COMPUTATIONAL OVERHEAD OF IMPROVED CCH1 AND IMPROVED CCH2 PROXY MULTI-SIGNATURE SCHEME}

\section{A. Review of improved CCH1 proxy multi-signature scheme}

There are four phases-Initialization phase, key generation phase, proxy multi-signature generation phase and proxy multi-signature verification phase [18].

Phase 1: Initialization phase over the elliptic curve domain following parameters must be known.

$>\quad$ A field size $\mathrm{p}$, which is odd prime.

$>$ Two parameters $\mathrm{a}, \mathrm{b}$ Fp to define the equation of elliptic curve $\mathrm{E}$ over Fp (i.e., y2 $=\mathrm{x} 3+\mathrm{ax}+$ $\mathrm{b}(\bmod \mathrm{p}))$, where $4 \mathrm{a} 3+27 \mathrm{~b} 2 \neq 0(\bmod \mathrm{p})$

$>$ A finite point $\mathrm{B}=(\mathrm{xB}, \mathrm{yB})$ whose order is a large prime number in $\mathrm{E}(\mathrm{Fp})$, where $\mathrm{B}$ is a point in $\mathrm{E}(\mathrm{Fp})$. Where $\mathrm{B} \neq \mathrm{O}$, because $\mathrm{O}$ denotes an infinity point.

$>\quad$ The order of $\mathrm{B}=\mathrm{t}$. 
Phase 2: Key generation phase: This phase is further divided into two parts.

Part 1: Personal key generation phase: All original signers and the designated proxy signer select their own individual secret keys.

- $\quad$ For each $1 \leq \mathrm{i} \leq \mathrm{n}$, the original signer $\mathrm{A}_{\mathrm{i}}$ secretly selects a random number $1 \leq \mathrm{d}_{\mathrm{i}} \leq \mathrm{t}-1$ as his private key, and computes the corresponding public key $\mathrm{Q}_{\mathrm{i}}=\mathrm{d}_{\mathrm{i}} \times \mathrm{B}=\left(\mathrm{x}_{\mathrm{Q}_{\mathrm{i}}}, \mathrm{y}_{\mathrm{Q}_{\mathrm{i}}}\right)$, where " $\times$ " indicates the multiplication of a number and elliptic curve point.

- The proxy signer is provided with a private key $1 \leq \mathrm{d}_{\mathrm{p}} \leq \mathrm{t}-1$ and a corresponding public key $\mathrm{Q}_{\mathrm{p}}$ $=\mathrm{d}_{\mathrm{p}} \times \mathrm{B}=\left(\mathrm{x}_{\mathrm{Qp}}, \mathrm{y}_{\mathrm{Qp}}\right)$.

Part 2: Proxy-signature secret key generation phase:

Step 1: (Secret key generation): For each $1 \leq \mathrm{i} \leq \mathrm{n}$, the original signer $\mathrm{A}_{\mathrm{i}}$ selects a random number $\mathrm{k}_{\mathrm{i}}$ $\{1,2 \ldots \mathrm{t}-1\} / \mathrm{d}_{\mathrm{i}}$ as secret key.

Step 2: (Group commitment value generation): Then computes

$\mathrm{R}_{\mathrm{i}}=\mathrm{k}_{\mathrm{i}} \times \mathrm{B}=\left(\mathrm{x}_{\mathrm{Ri}}, \mathrm{y}_{\mathrm{Ri}}\right)$

If $\mathrm{x}_{\mathrm{Ri}}=0$ then return to step 1 ; otherwise $A_{i}$ gives $\mathrm{R}_{\mathrm{i}}$ to other original signers.

Step 3: (Sub-delegation parameter generation): For each $1 \leq \mathrm{i} \leq \mathrm{n}$, the original signer $\mathrm{A}_{\mathrm{i}}$ uses his own secret keys $d_{i}, k_{i}$ and the group commitment value $\mathrm{x}_{\mathrm{R}}$ to compute the following:

$\mathrm{s}_{\mathrm{i}}=\mathrm{d}_{\mathrm{i}} * \mathrm{x}_{\mathrm{Q}_{\mathrm{i}}} * \mathrm{~h}\left(\mathrm{M}_{\mathrm{w}}, \mathrm{R}_{\mathrm{i}}\right)-\mathrm{k}_{\mathrm{i}} * \mathrm{x}_{\mathrm{R}_{\mathrm{i}}}(\bmod \mathrm{t})$

Where $\mathrm{h} \mathrm{(} \mathrm{)} \mathrm{is} \mathrm{a} \mathrm{hash} \mathrm{function} \mathrm{and} \mathrm{the} \mathrm{warrant}$ $\mathrm{M}_{\mathrm{w}}$ contains information such as the IDs of all original signers and proxy signer. Then, the sub-delegation parameter for $A_{i}$ is $\left(M_{w}, R_{i}, s_{i}\right)$.

Step 4: (Sub-delegation parameter verification): After the proxy signer has received the subdelegation parameters then the proxy signer $\mathrm{P}$ computes

$\mathrm{S}_{\mathrm{i}} \times \mathrm{B}=\left(\mathrm{x}_{\mathrm{Q}_{\mathrm{i}}} * \mathrm{~h}\left(\mathrm{M}_{\mathrm{w}}, \mathrm{R}_{\mathrm{i}}\right)\right) * \mathrm{Q}_{\mathrm{i}}-\mathrm{x}_{\mathrm{R}_{\mathrm{i}}} \mathrm{R}_{\mathrm{i}}$

and check whether it holds. If it holds then the proxy signer accepts $\left(\mathrm{M}_{\mathrm{w}}, \mathrm{R}_{\mathrm{i}}, \mathrm{s}_{\mathrm{i}}\right)$ as a valid subdelegation parameter; otherwise, he can reject it and requests a valid one $A_{i}$, or terminate this protocol.

$\underline{\text { Step } 5}$ :( Proxy multi-signature secret key generation): Then computes the proxy multi-signature secret key as follows:

$\mathrm{d}=\mathrm{dp}^{*} \mathrm{xQp}+\sum \mathrm{ni}=1 \mathrm{si} \bmod \mathrm{t}$

Phase 3: Proxy multi-signature generation phase: The proxy multi-signature is in the form of $(\mathrm{m}, \mathrm{mw}, \mathrm{R}$,
$\operatorname{Sigd}(\mathrm{m}))$, where $\operatorname{Sigd}(\mathrm{m})$ is the signature generated by a designated signature scheme (EC-schnorr signature scheme) using the proxy signing key $\mathrm{d}$ and $\mathrm{m}$ is message.

Step 1: Proxy signer $P$ choose random number $\mathrm{j}$ where $1 \leq \mathrm{j} \leq \mathrm{t}-1$ and calculate $\mathrm{J}=\mathrm{j} \times \mathrm{B}=\left(\mathrm{J}_{\mathrm{x}}, \mathrm{J}_{\mathrm{y}}\right)$.

Step 2: Compute $\mathrm{e}=\mathrm{h}\left(\mathrm{m}, \mathrm{J}_{\mathrm{x}}\right)$ where $\mathrm{h}\left(\mathrm{J}_{\mathrm{x}}, \mathrm{m}\right)$ is hash function. If $\mathrm{e}=0$ then go to step 1 .

Step 3: Compute

$$
\mathrm{y}=\mathrm{j}-\mathrm{d} * \mathrm{e}(\bmod \mathrm{t})
$$

and the output $\operatorname{sign}_{\mathrm{d}}(\mathrm{m})=(\mathrm{e}, \mathrm{y})$.

Phase 4: Proxy multi-signature verification phase: When the verifier verifies the signature, he or she calculates the proxy public value Q corresponding to the proxy signature key d as

$\mathrm{Q}=\mathrm{Q}_{\mathrm{P}} * \mathrm{x}_{\mathrm{Q}_{\mathrm{p}}}+\sum_{\mathrm{i}=1}^{\mathrm{n}}\left(\mathrm{h}\left(\mathrm{M}_{\mathrm{w}}, \mathrm{R}_{\mathrm{i}}\right) * \mathrm{Q}_{\mathrm{i}}-\mathrm{x}_{\mathrm{R}_{\mathrm{i}}} \times \mathrm{R}_{\mathrm{i}}\right)$

With the value, the verifier can confirm the validity of $\mathrm{Sig}_{\mathrm{d}}(\mathrm{m})$ by validating the verification equality of the designated signature scheme.

\section{$\underline{\text { Step 1: }}$ Compute $\overline{\mathrm{J}}=\mathrm{y} \times \mathrm{B}+\mathrm{e} \times \mathrm{Q}=\left(\overline{\mathrm{J}}_{\mathrm{x}}, \overline{\mathrm{J}}_{\mathrm{V}}\right)$}

Step 2: And compute $\mathrm{e}^{\prime}=\mathrm{h}\left(\mathrm{J}_{\mathrm{x}}, \mathrm{m}\right)$. Then check that $\mathrm{e}^{\prime}=$ $\mathrm{e}$ and if these are equal then valid signature otherwise not.

B. Review of improved $\mathrm{CCH} 2$ proxy multi-signature scheme

There are four phases-Initialization phase, key generation phase, proxy multi-signature generation phase and proxy multi-signature verification phase [18].

Phase 1: Initialization phase: Over the elliptic curve domain following parameters must be known.

$>$ A field size $\mathrm{p}$, which is a odd prime.

$>$ Two parameters $\mathrm{a}, \mathrm{b}$ Fp to define the equation of elliptic curve $\mathrm{E}$ over Fp (i.e., $\mathrm{y} 2=\mathrm{x} 3+\mathrm{ax}+$ $\mathrm{b}(\bmod \mathrm{p}))$, where $4 \mathrm{a} 3+27 \mathrm{~b} 2 \neq 0(\bmod \mathrm{p})$

$>$ A finite point $\mathrm{B}=(\mathrm{xB}, \mathrm{yB})$ whose order is a large prime number in $\mathrm{E}(\mathrm{Fp})$, where $\mathrm{B}$ is a point in $\mathrm{E}(\mathrm{Fp})$. Where $\mathrm{B} \neq \mathrm{O}$, because $\mathrm{O}$ denotes an infinity point.

$>$ The order of $\mathrm{B}=\mathrm{t}$.

Phase 2: Key generation phase: This phase is further divided into two parts:

Part 1: Personal key generation phase: All original signers and the designated proxy signer are authorized to select their own individual secret keys.

- $\quad$ For each $1 \leq \mathrm{i} \leq \mathrm{n}$, the original signer $\mathrm{A}_{\mathrm{i}}$ secretly selects a random number $1 \leq \mathrm{d}_{\mathrm{i}} \leq \mathrm{t}-1$ as his 
private key, and computes the corresponding public key $\mathrm{Q}_{\mathrm{i}}=\mathrm{d}_{\mathrm{i}} \times \mathrm{B}=\left(\mathrm{x}_{\mathrm{Q}_{\mathrm{i}}}, \mathrm{y}_{\mathrm{Q}_{\mathrm{i}}}\right)$, where " $\times$ " indicates the multiplication of a number by an elliptic curve point.

- The proxy signer is provided with a private key $1 \leq \mathrm{d}_{\mathrm{p}} \leq \mathrm{t}-1$ and a corresponding public key $\mathrm{Q}_{\mathrm{p}}$ $=\mathrm{d}_{\mathrm{p}} \times \mathrm{B}=\left(\mathrm{x}_{\mathrm{Qp}}, \mathrm{y}_{\mathrm{Qp}}\right)$. All public keys $\mathrm{Q}_{\mathrm{i}}$ and $\mathrm{Q}_{\mathrm{p}}$ must be certified by the $\mathrm{CA}$.

Part 2: Proxy-signature secret key generation phase:

Step 1: (Secret key generation): For each $1 \leq \mathrm{i} \leq \mathrm{n}$, the original signer $A_{i}$ selects a random number $k_{i}$ $\{1,2 \ldots \mathrm{t}-1\} / \mathrm{d}_{\mathrm{i}}$ as secret key.

Step 2: (Group commitment value generation): Then computes $\mathrm{R}_{\mathrm{i}}=\mathrm{k}_{\mathrm{i}} \times \mathrm{B}=\left(\mathrm{x}_{\mathrm{Ri}}, \mathrm{y}_{\mathrm{Ri}}\right)$. If $\mathrm{x}_{\mathrm{Ri}}=0$ then return to step 1; otherwise $A_{i}$ broadcasts $R_{i}$ to other original signers. On receiving $R_{j}(1 \leq j \leq n$, $\mathrm{j} \neq \mathrm{i}), \mathrm{A}_{\mathrm{i}}$ calculates

$R_{\mathrm{i}}=\left(\mathrm{x}_{\mathrm{R}}, \mathrm{y}_{\mathrm{R}}\right)$

Step 3: (Sub-delegation parameter generation): For each $1 \leq \mathrm{i} \leq \mathrm{n}$, the original signer $\mathrm{A}_{\mathrm{i}}$ uses his own secret keys $d_{i}, k_{i}$ and the group commitment value $\mathrm{x}_{\mathrm{R}}$ to compute the following.

$\mathrm{s}_{\mathrm{i}}=\mathrm{d}_{\mathrm{i}} * \mathrm{~h}\left(\mathrm{M}_{\mathrm{w}}, \mathrm{x}_{\mathrm{Qp}}, \mathrm{x}_{\mathrm{Qi}}, \mathrm{x}_{\mathrm{R}}\right)-\mathrm{k}_{\mathrm{i}} * \mathrm{x}_{\mathrm{R}}(\bmod \mathrm{t})$

Where $\mathrm{h}(\mathrm{)}$ is a hash function and the warrant $\mathrm{M}_{\mathrm{w}}$ contains information such as the IDs of all original signers and proxy signer. Then, the sub-delegation parameter for $A_{i}$ is $\left(M_{w}, s_{i}\right)$.

Step 4: (Sub-delegation parameter verification): After the proxy signer has received the subdelegation parameters then the proxy signer $\mathrm{P}$ computes

si * B = h(Mw, xQp ,xQi , xR) *Qi - xR × Ri

checks whether it holds. If it holds then the proxy signer accepts $\left(\mathrm{M}_{\mathrm{w}}, \mathrm{s}_{\mathrm{i}}\right)$ as a valid subdelegation parameter; otherwise, he can reject it and requests a valid one $A_{i}$, or terminate this protocol.

$\underline{\text { Step } 5}$ :( Proxy multi-signature secret key generation): Then computes the proxy multi-signature secret key as follows:

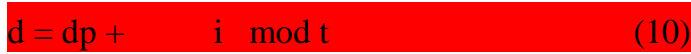

Phase 3: Proxy multi-signature generation phase: The proxy multi-signature is in the form of $(\mathrm{m}, \mathrm{mw}, \mathrm{R}, \operatorname{Sigd}(\mathrm{m}))$, where $\operatorname{Sigd}(\mathrm{m})$ is the signature generated by a designated signature scheme (EC-schnorr signature scheme) using the proxy signing key $\mathrm{d}$ and $\mathrm{m}$ is message.
Step 1: Proxy signer $P$ choose random number $\mathrm{j}$ where $1 \leq \mathrm{j} \leq \mathrm{t}-1$ and calculate $\mathrm{J}=\mathrm{j} \times \mathrm{B}=\left(\mathrm{J}_{\mathrm{x}}, \mathrm{J}_{\mathrm{y}}\right)$.

Step 2: Compute $\mathrm{e}=\mathrm{h}\left(\mathrm{m}, \mathrm{J}_{\mathrm{x}}\right)$ where $\mathrm{h}\left(\mathrm{J}_{\mathrm{x}}, \mathrm{m}\right)$ is hash function. If $\mathrm{e}=0$ then go to step 1 .

Step 3: Compute

$$
\begin{aligned}
& y=j-d \times e(\bmod t) \\
& \text { and the output } \operatorname{sign}_{d}(m)=(e, y)
\end{aligned}
$$

Phase 4: Proxy multi-signature verification phase: When the verifier verifies the signature, he or she calculates the proxy public value Q corresponding to the proxy signature key $\mathrm{d}$ as

$$
\mathrm{Q}=\mathrm{Q}_{\mathrm{P}}+\left(\mathrm{M}_{\mathrm{w}}, \mathrm{x}_{\mathrm{Qp}}, \mathrm{x}_{\mathrm{Qi}}, \mathrm{x}_{\mathrm{R}}\right) \times \mathrm{Q}_{\mathrm{i}}-\mathrm{R} \times \mathrm{x}_{\mathrm{R}}
$$

With the value, the verifier can confirm the validity of $\operatorname{Sig}_{d}(\mathrm{~m})$ by validating the verification equality of the designated signature scheme.

\section{Step 1: Compute}

Step 2: And compute $\mathrm{e}^{\prime}=\mathrm{h}\left(\overline{\mathrm{J}}_{\mathrm{x}}, \mathrm{m}\right)$. Then check that $\mathrm{e}^{\prime}=$ $\mathrm{e}$ and if these are equal then valid signature otherwise not.

C. Time, Space and Computational overhead of improved $\mathrm{CCH} 1$ and $\mathrm{CCH} 2$ scheme

This table shows the time, space and computational overhead of improved $\mathrm{CCH} 1$ and $\mathrm{CCH} 2$ proxy multisignature scheme with varying value of field size $(p)$.

TABLE NO. 1 Time, space and computational overhead of improved $\mathrm{CCH} 1$ and $\mathrm{CCH} 2$ scheme

\begin{tabular}{|c|c|c|c|c|c|c|c|}
\hline $\begin{array}{c}\text { Sr. } \\
\text { No }\end{array}$ & $p$ & \multicolumn{2}{|c|}{$\begin{array}{c}\text { Time } \\
\text { complexity }\end{array}$} & \multicolumn{2}{c|}{$\begin{array}{c}\text { Space } \\
\text { complexity }\end{array}$} & \multicolumn{2}{c|}{$\begin{array}{c}\text { Computational } \\
\text { overhead }\end{array}$} \\
\hline & & CCH1 & CCH2 & CCH1 & CCH2 & CCH1 & CCH2 \\
\hline 1. & 5 & 1724 & 1549 & 1994 & 1810 & 211 & 175 \\
\hline 2. & 7 & 1619 & 1358 & 1894 & 1379 & 208 & 177 \\
\hline 3. & 11 & 1786 & 1369 & 1641 & 1515 & 210 & 180 \\
\hline 4. & 13 & 1503 & 1494 & 1565 & 1459 & 211 & 168 \\
\hline 5. & 17 & 1583 & 1426 & 1813 & 1524 & 210 & 171 \\
\hline 6. & 19 & 1777 & 1461 & 1774 & 1308 & 211 & 169 \\
\hline 7. & 23 & 1728 & 1572 & 1905 & 1505 & 218 & 177 \\
\hline 8. & 29 & 1761 & 1456 & 1979 & 1629 & 212 & 187 \\
\hline 9. & 31 & 1748 & 1448 & 1914 & 1602 & 213 & 170 \\
\hline 10. & 37 & 1590 & 1419 & 1918 & 1700 & 203 & 184 \\
\hline 11. & 41 & 1510 & 1407 & 1576 & 1524 & 213 & 187 \\
\hline 12. & 43 & 1535 & 1516 & 1624 & 1405 & 215 & 175 \\
\hline 13. & 47 & 1745 & 1359 & 1517 & 1387 & 204 & 168 \\
\hline 14. & 53 & 1539 & 1350 & 1963 & 1506 & 203 & 182 \\
\hline 15. & 59 & 1613 & 1431 & 1934 & 1508 & 215 & 172 \\
\hline 16. & 61 & 1624 & 1421 & 1987 & 1644 & 208 & 181 \\
\hline 17. & 67 & 1581 & 1350 & 1695 & 1661 & 217 & 170 \\
\hline 18. & 71 & 1636 & 1340 & 1964 & 1391 & 216 & 183 \\
\hline 19. & 73 & 1667 & 1391 & 1744 & 1648 & 217 & 175 \\
\hline 20. & 79 & 1779 & 1418 & 1813 & 1405 & 214 & 185 \\
\hline 21. & 83 & 1554 & 1363 & 1943 & 1464 & 208 & 173 \\
\hline 22. & 89 & 1583 & 1575 & 1904 & 1515 & 209 & 176 \\
\hline 23. & 97 & 1553 & 1438 & 1927 & 1517 & 219 & 184 \\
\hline & & & & & & & \\
\hline
\end{tabular}


D. Graphical representation of Time, Space and Computational overhead of improved $\mathrm{CCH} 1$ and $\mathrm{CCH} 2$ scheme

Graphs shows the time, space and computational overhead of improved $\mathrm{CCH} 1$ and $\mathrm{CCH} 2$ proxy multisignature scheme with varying value of field size $(\mathrm{p})$.

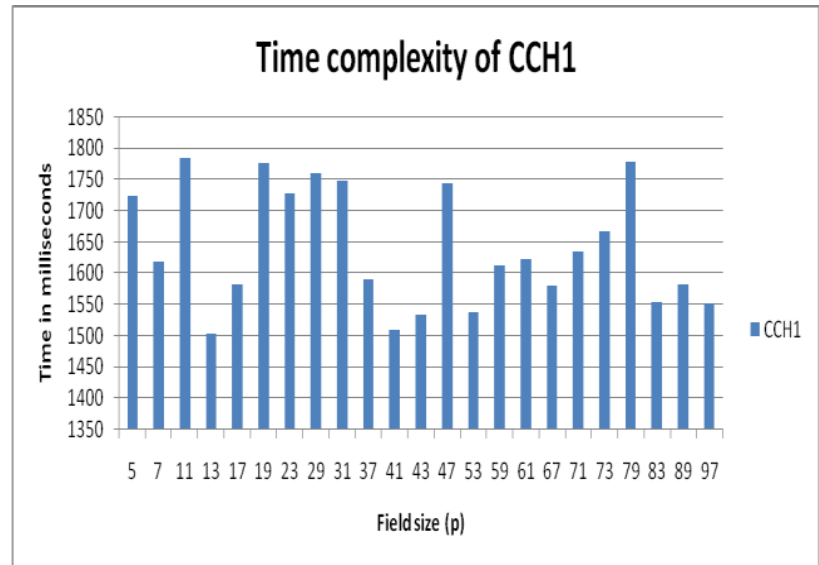

Figure. No. 1 Time complexity of improved CCH1 scheme with varying value of field size $(\mathrm{p})$

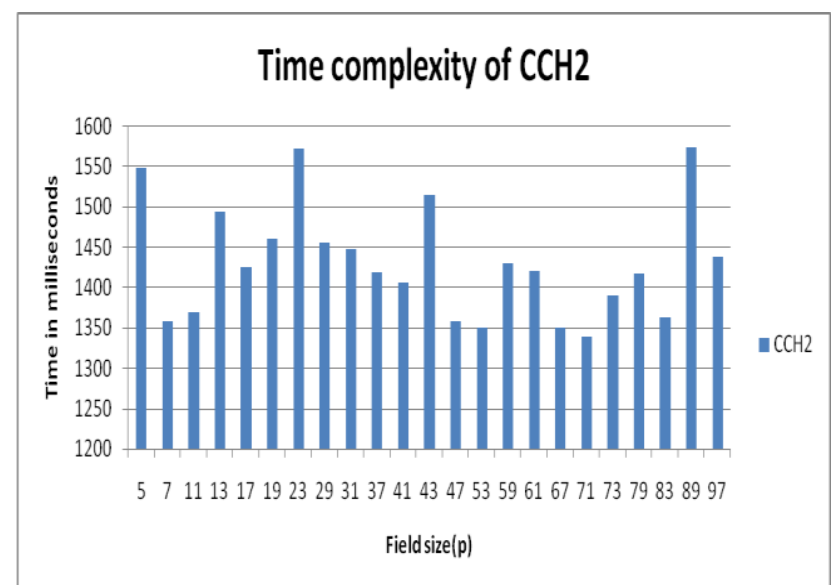

Figure. No. 2 Time complexity of improved $\mathrm{CCH} 2$ scheme with varying value of field size $(p)$

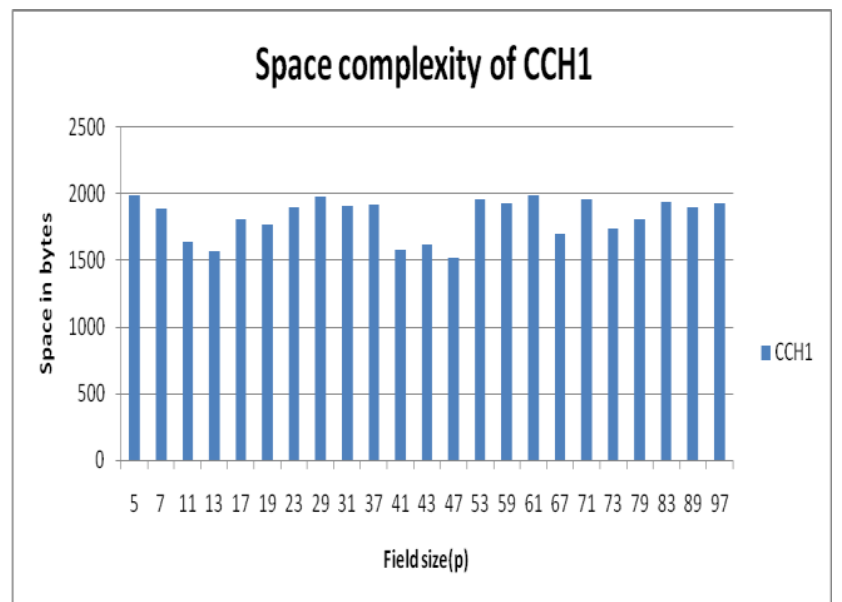

Figure. No. 3 Space complexity of improved CCH1 scheme with varying value of field size $(p)$

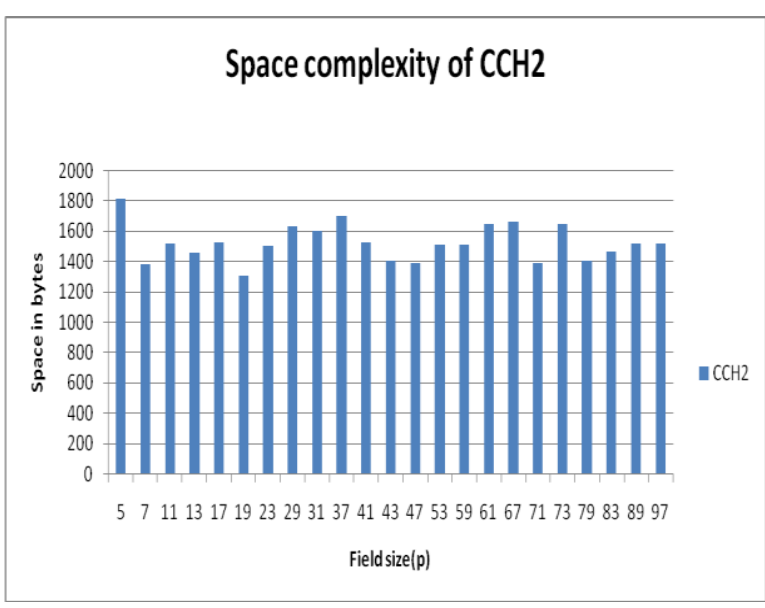

Figure. No. 4 Space complexity of improved CCH2 scheme with varying value of field size $(\mathrm{p})$

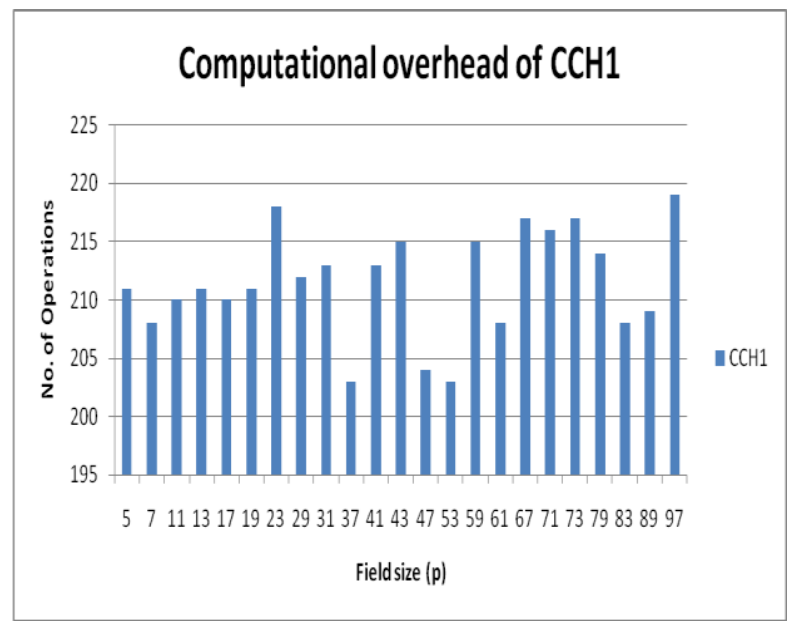

Figure. No. 5 Computational overhead of improved CCH1 scheme with varying value of field size (p)

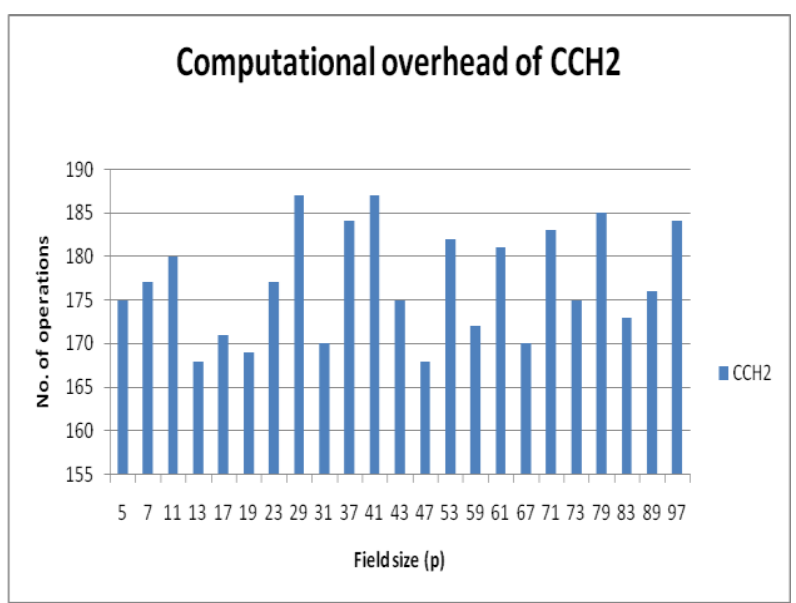

Figure. No. 6 Computational overhead of improved CCH2 scheme with varying value of field size $(\mathrm{p})$

\section{CRYPTANALYSIS OF IMPROVED CCH2 PROXY MULTI-SIGNATURE SCHEME}

Elliptic curve based schnorr signature scheme is used in improved $\mathrm{CCH} 2$ proxy multi-signature scheme to generate and verify the signature. 


\section{A. Forge a proxy multi-signature}

Suppose proxy signer $\mathrm{P}$ signed a message with his private key dp and the signature that are generated is $(\mathrm{m}$, $\left.\mathrm{mw}, \mathrm{R}, \mathrm{e}, \mathrm{y}^{\prime \prime}\right)$ where $\mathrm{e}=\mathrm{h}(\mathrm{m}, \mathrm{jx})$ and $\mathrm{y}^{\prime \prime}=\mathrm{j}-\mathrm{dp} * \mathrm{e}(\mathrm{mod}$ t). Upon received the signature $\left(\mathrm{m}, \mathrm{mw}, \mathrm{R}, \mathrm{e}, \mathrm{y}^{\prime \prime}\right)$ the malicious original signers A1...An can forge proxy signature by computing following operations:

- $\quad$ Compute $\mathrm{y}^{\prime}=\sum_{i=1}^{n} s_{\mathrm{i}} *$ e $\bmod \mathrm{t}$.

- Compute $\mathrm{y}=\mathrm{y}^{\prime \prime}-\mathrm{y}^{\prime}$

Finally, the malicious original signers A1...An can forge proxy signature $(\mathrm{m}, \mathrm{mw}, \mathrm{R}, \mathrm{e}, \mathrm{y})$. The following proof shows that why the proxy signature $(\mathrm{m}, \mathrm{mw}, \mathrm{R}, \mathrm{e}$, y) is valid.

PROFF:

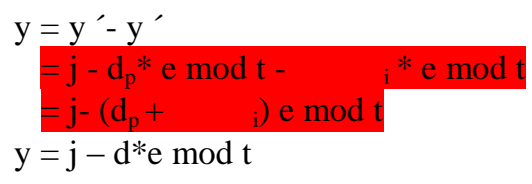

Proxy multi-signature verification: When the verifier verifies the signature, he or she calculates the proxy public value $\mathrm{Q}$ corresponding to the proxy signature key d as

Then, the verifier can confirm the validity of Sigd (m) by validating the verification equality of the designated signature scheme.

\section{Step 1: Compute $\overline{\mathrm{J}}=\mathrm{y} \times \mathrm{B}+\mathrm{e} \times \mathrm{O}=\left(\overline{\mathrm{J}}_{\mathrm{x}}, \overline{\mathrm{J}}_{\mathrm{y}}\right)$}

Step 2: And compute $\mathrm{e}^{\prime}=\mathrm{h}\left(\mathrm{J}_{\mathrm{x}}, \mathrm{m}\right)$. Then check that $\mathrm{e}^{\prime}=$ $\mathrm{e}$ and if these are equal then valid signature otherwise not.

\section{B. Forge the proxy signer's signature}

After getting signature $(\mathrm{m}, \mathrm{mw}, \mathrm{R}, \mathrm{e}, \mathrm{y})$ that are generated by proxy signer on behalf of original signers Where $e=h(m, j x)$ and $y=j-d * e(\bmod t)$, the original signer $\mathrm{A} 1$...An can forge proxy signer P's signature on message $\mathrm{m}$ by computing following opeartions:

- $\quad$ Each $\mathrm{A}_{\mathrm{i}}$ compute $\mathrm{s}_{\mathrm{i}}$ * e where $\mathrm{i}=1,2, . . \mathrm{n}$.

- Compute $\mathrm{y}^{\prime}=\sum_{i=1}^{n} s_{\mathrm{i}} *$ e $\bmod \mathrm{t}$.

- Compute $\mathrm{y}^{\prime}=\mathrm{y}+\mathrm{y}^{\prime}$.

- $\left(\mathrm{m}, \mathrm{m}_{\mathrm{w}}, \mathrm{R}, \mathrm{e}, \mathrm{y}^{\prime \prime}\right)$ is valid signature on message $\mathrm{m}$.

The malicious original signers can forge signature $(\mathrm{m}$, $\mathrm{mw}, \mathrm{R}, \mathrm{e}, \mathrm{y}^{\prime \prime}$ ) on message $\mathrm{m}$ with respect to proxy signer P's private key dp.

The following proof shows that why the proxy signature $\left(\mathrm{m}, \mathrm{mw}, \mathrm{R}, \mathrm{e}, \mathrm{y}^{\prime \prime}\right)$ is valid

PROFF:

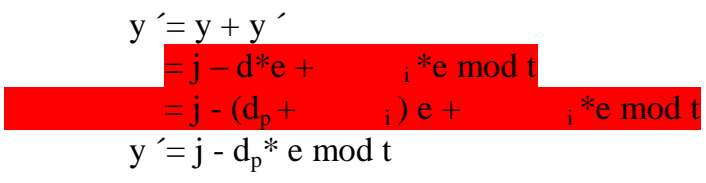

Proxy multi-signature verification: When the verifier verifies the signature, he or she use proxy public value Qp corresponding to the proxy signature key dp. Then the verifier can confirm the validity of Sigd (m) by validating the verification equality of the designated signature scheme.

\section{Step 1: Compute $\overline{\mathrm{J}}=\mathrm{y} \times \mathrm{B}+\mathrm{e} \times \mathrm{Q}_{\mathrm{p}}=\left(\bar{J}_{\mathrm{x}}, \bar{J}_{\mathrm{y}}\right)$}

Step 2: And compute $\mathrm{e}^{\prime}=\mathrm{h}\left(\overline{\mathrm{J}}_{\mathrm{x}}, \mathrm{m}\right)$. Then check that $\mathrm{e}^{\prime}=$ $\mathrm{e}$ and if these are equal then valid signature otherwise not.

\section{Framing Attack}

On behalf of users A1, A2 ... An, malicious users A1, A2 ... An can forge a proxy multi-signature for message $\mathrm{m}$ by some user $\mathrm{P}$, such that user $\mathrm{P}$ was never designated by users A1,A2 ...An. Suppose proxy signer P signed a message with his private key dp, the signature is $(\mathrm{m}, \mathrm{mw}$, $\left.\mathrm{R}, \mathrm{e}, \mathrm{y}^{\prime}\right)$ where $\mathrm{e}=\mathrm{h}(\mathrm{m}, \mathrm{jx})$ and $\mathrm{y}^{\prime \prime}=\mathrm{j}-\mathrm{dp} * \mathrm{e}(\bmod \mathrm{t})$.

Upon received the signature $\left(\mathrm{m}, \mathrm{mw}, \mathrm{R}, \mathrm{e}, \mathrm{y}^{\prime \prime}\right)$, the malicious original signers A1...An can forge proxy multi-signature by performing following steps:

- $\quad$ The malicious users $A_{1}, A_{2} \ldots A_{n}$ pretend to produce a forge warrant $\mathrm{m}_{\mathrm{w}}$, which recording the delegation information such as identities of the malicious users $\mathrm{A}_{1}, \mathrm{~A}_{2} \ldots \mathrm{A}_{\mathrm{n}}$ and user $\mathrm{P}$.

- For each $1 \leq \mathrm{i} \leq \mathrm{n}$, the malicious user $\mathrm{A}_{\mathrm{i}}$ selects a random number $1 \leq k_{i} \leq t-1$, and then computes $R_{i}$ $=\mathrm{k}_{\mathrm{i}} \mathrm{X} \mathrm{B}=\left(\mathrm{x}_{\mathrm{Ri}} \mathrm{y}_{\mathrm{Ri}}\right)$ and broadcast $\mathrm{R}_{\mathrm{i}}$ to other users.

- $\quad$ On receiving $R_{j}(1 \leq j \leq n, j \neq i), A_{i}$ calculates

$$
\begin{aligned}
& \mathrm{R}=\sum_{\mathrm{i}=1}^{\mathrm{n}} \mathrm{R}_{\mathrm{i}}=\left(\mathrm{x}_{\mathrm{R}}, \mathrm{y}_{\mathrm{R}}\right) \\
& \mathrm{s}_{\mathrm{i}}=\mathrm{d}_{\mathrm{i}} * \mathrm{~h}\left(\mathrm{M}_{\mathrm{w}}, \mathrm{x}_{\mathrm{Q}_{\mathrm{p}},} \mathrm{x}_{\mathrm{Q}_{\mathrm{i}}} \mathrm{x}_{\mathrm{R}}\right)-\mathrm{k}_{\mathrm{i}} * \mathrm{x}_{\mathrm{R}}(\bmod \mathrm{t})
\end{aligned}
$$

Note that user $\mathrm{P}$ doesn't receive any information from the malicious users A1, A2...An.

- $\quad$ Compute $\mathrm{y}^{\prime}=\sum_{i=1}^{n} s_{\mathrm{i}} * \mathrm{e} \bmod \mathrm{t}$;

- Compute $\mathrm{y}=\mathrm{y}^{\prime \prime}-\mathrm{y}^{\prime}$

Finally the malicious users can forge a valid signature ( $\mathrm{m}, \mathrm{mw}, \mathrm{R}, \mathrm{e}, \mathrm{y})$ on message $\mathrm{m}$ by some user $\mathrm{P}$ on behalf of users $\mathrm{A} 1 \ldots \mathrm{An}$, such that user $\mathrm{P}$ was never designated by users A1...An. The following shows why the signature $(\mathrm{m}, \mathrm{mw}, \mathrm{R}, \mathrm{e}, \mathrm{y})$ is valid.

PROFF:

$$
\begin{aligned}
\mathrm{y} & =\mathrm{y}^{\prime \prime}-\mathrm{y}^{\prime} \\
& =\mathrm{j}-\mathrm{d}_{\mathrm{p}} * \mathrm{e} \bmod \mathrm{t}-\sum_{\mathrm{i}=1}^{n} s_{\mathrm{i}} * \mathrm{e} \bmod \mathrm{t} \\
& =\mathrm{j}-\left(\mathrm{d}_{\mathrm{p}}+\sum_{\mathrm{i}=1}^{n} s_{\mathrm{i}}\right) \mathrm{e} \bmod \mathrm{t} \\
\mathrm{y} & =\mathrm{j}-\mathrm{d}^{*} \mathrm{e} \bmod \mathrm{t}
\end{aligned}
$$


From above we can see that the malicious users A1...An framed innocent user P.

Proxy multi-signature verification phase: When the verifier verifies the signature, he or she calculates the proxy public value $\mathrm{Q}$ corresponding to the proxy signature key d as

$\mathrm{Q}=\mathrm{Q}_{\mathrm{P}}+\quad\left(\mathrm{M}_{\mathrm{w}}, \mathrm{x}_{\mathrm{Op}}, \mathrm{x}_{\mathrm{Oi}}, \mathrm{x}_{\mathrm{R}}\right) \times \mathrm{Q}_{\mathrm{i}}-\mathrm{R} \times \mathrm{x}_{\mathrm{R}}$

With the value, the verifier can confirm the validity of Sigd $(\mathrm{m})$ by validating the verification equality of the designated signature scheme.

\section{Step 1: Compute}

Step 2: And compute $\mathrm{e}^{\prime}=\mathrm{h}\left(\mathrm{J}_{\mathrm{x}}, \mathrm{m}\right)$. Then check that $\mathrm{e}^{\prime}=$ $\mathrm{e}$ and if these are equal then valid signature otherwise not.

\section{ENHANCED PROXY MULTI-SIGNATURE SCHEME BASED ON ECC}

There are four phases-Initialization phase, key generation phase, proxy multi-signature generation phase and proxy multi-signature verification phase.

Phase 1: System initialization phase: Before the whole scheme can be initialized, the following parameters over the elliptic curve domain must be known.

$>\quad$ A field size $\mathrm{p}$, which is a large odd prime.

$>$ Two parameters a, b Fp to define the equation of elliptic curve $\mathrm{E}$ over Fp (i.e., y2 = $\mathrm{x} 3+\mathrm{ax}+\mathrm{b}(\bmod \mathrm{p}))$, where $4 \mathrm{a} 3+27 \mathrm{~b} 2 \neq 0$ $(\bmod \mathrm{p})$

$>$ A finite point $\mathrm{B}=(\mathrm{xB}, \mathrm{yB})$ whose order is a large prime number in $\mathrm{E}(\mathrm{Fp})$, where $\mathrm{B}$ is a point in $\mathrm{E}(\mathrm{Fp})$. Where $\mathrm{B} \neq \mathrm{O}$, because $\mathrm{O}$ denotes an infinity point.

$>$ The order of $\mathrm{B}=\mathrm{t}$.

Phase 2: Key generation phase: This phase can be further divided into two parts.

Part 1: Personal public key generation phase: All original signers and the designated proxy signer are authorized to select their own individual secret keys.

- $\quad$ For each $1 \leq \mathrm{i} \leq \mathrm{n}$, the original signer $\mathrm{A}_{\mathrm{i}}$ secretly selects a random number $1 \leq \mathrm{d}_{\mathrm{i}} \leq \mathrm{t}-1$ as his private key, and computes the corresponding public key $\mathrm{Q}_{\mathrm{i}}=\mathrm{d}_{\mathrm{i}} \times \mathrm{B}=\left(\mathrm{x}_{\mathrm{Q}_{\mathrm{i}}}, \mathrm{y}_{\mathrm{Q}_{\mathrm{i}}}\right)$, where “ $\times$ " indicates the multiplication of a number by an elliptic curve point.

- The proxy signer is provided with a private key $1 \leq \mathrm{d}_{\mathrm{p}} \leq \mathrm{t}-1$ and a corresponding public key $\mathrm{Q}_{\mathrm{p}}$ $=\mathrm{d}_{\mathrm{p}} \times \mathrm{B}=\left(\mathrm{x}_{\mathrm{Qp}}, \mathrm{y}_{\mathrm{Qp}}\right)$. All public keys $\mathrm{Q}_{\mathrm{i}}$ and $\mathrm{Q}_{\mathrm{p}}$ must be certified by the $\mathrm{CA}$.

Part 2: Proxy-signature secret key generation phase:
Step 1: (Secret key generation): For each $1 \leq \mathrm{i} \leq \mathrm{n}$, the original signer $A_{i}$ selects a random number $k_{i}$ $\{1,2 \ldots t-1\} / d_{i}$ as secret key.

Step 2: (Group commitment value generation): Then computes $\mathrm{R}_{\mathrm{i}}=\mathrm{k}_{\mathrm{i}} \times \mathrm{B}=\left(\mathrm{x}_{\mathrm{Ri}}, \mathrm{y}_{\mathrm{Ri}}\right)$. If $\mathrm{x}_{\mathrm{Ri}}=0$ then return to step 1; otherwise $A_{i}$ broadcasts $R_{i}$ to other original signers. On receiving $R_{j}(1 \leq j \leq n$, $\mathrm{j} \neq \mathrm{i}), \mathrm{A}_{\mathrm{i}}$ calculates

Step 3: (Sub-delegation parameter generation): For each $1 \leq \mathrm{i} \leq \mathrm{n}$, the original signer $\mathrm{A}_{\mathrm{i}}$ uses his own secret keys $d_{i}, k_{i}$ and the group commitment value $x_{R}$ to compute the following.

$\mathrm{s}_{\mathrm{i}}=\mathrm{d}_{\mathrm{i}} * \mathrm{~h}\left(\mathrm{M}_{\mathrm{w}}, \mathrm{x}_{\mathrm{Qp}}, \mathrm{x}_{\mathrm{Qi}}, \mathrm{x}_{\mathrm{R}}\right)-\mathrm{k}_{\mathrm{i}} * \mathrm{x}_{\mathrm{R}}(\bmod \mathrm{t})$

Where $\mathrm{h}(\mathrm{)}$ is a public collision-resistant hash function and the warrant $\mathrm{M}_{\mathrm{w}}$ contains information such as the IDs of all original signers and proxy signer, and the delegation period. Then, the sub-delegation parameter for $\mathrm{A}_{\mathrm{i}}$ is $\left(\mathrm{M}_{\mathrm{w}}, \mathrm{s}_{\mathrm{i}}\right)$.

Step 4: (Sub-delegation parameter delivery): For each $1 \leq \mathrm{i} \leq \mathrm{n}$, the original signer $\mathrm{A}_{\mathrm{i}}$ sends $\left(\mathrm{M}_{\mathrm{w}}, \mathrm{s}_{\mathrm{i}}\right)$ to the proxy signer via a public channel.

Step 5: (Sub-delegation parameter verification): After the proxy signer has received the subdelegation parameters then the proxy signer $\mathrm{P}$ computes

$\mathrm{s}_{\mathrm{i}} * \mathrm{~B}=\mathrm{h}\left(\mathrm{M}_{\mathrm{w}}, \mathrm{x}_{\mathrm{Qp}}, \mathrm{x}_{\mathrm{Qi}}, \mathrm{x}_{\mathrm{R}}\right) * \mathrm{Q}_{\mathrm{i}}-\mathrm{x}_{\mathrm{R}} * \mathrm{R}_{\mathrm{i}}$

Checks whether it holds. If it holds then theproxy signer accepts $\left(\mathrm{M}_{\mathrm{w}}, \mathrm{s}_{\mathrm{i}}\right)$ as a valid subdelegation parameter; otherwise, he can reject it and requests a valid one $A_{i}$, or terminate this protocol.

Step 6 : (Proxy multi-signature secret key generation): Then computes the proxy multi-signature secret key as follows:

\section{$\mathrm{d}=\mathrm{x}_{\mathrm{Q}_{\mathrm{p}}} * \mathrm{~d}_{\mathrm{p}}+\quad \mathrm{i} \bmod \mathrm{t}$}

Phase 3: Proxy multi-signature generation phase: The proxy multi-signature affixed to the $\mathrm{m}$ is in the form of $(\mathrm{m}, \mathrm{mw}, \mathrm{R}, \operatorname{Sigd}(\mathrm{m}))$, where $\operatorname{Sigd}(\mathrm{m})$ is the signature generated by a designated signature scheme (EC-schnorr signature scheme) using the proxy signing key $\mathrm{d}$ and $\mathrm{m}$ is message.

Step 1: Proxy signer $P$ choose random number $\mathrm{j}$ where $1 \leq \mathrm{j} \leq \mathrm{t}-1$ and calculate $\mathrm{J}=\mathrm{j} \times \mathrm{B}=\left(\mathrm{J}_{\mathrm{x}}, \mathrm{J}_{\mathrm{y}}\right)$.

Step 2: Compute $\mathrm{e}=\mathrm{h}\left(\mathrm{m}, \mathrm{J}_{\mathrm{x}}\right)$ where $\mathrm{h}\left(\mathrm{J}_{\mathrm{x}}, \mathrm{m}\right)$ is hash function. If $\mathrm{e}=0$ then go to step 1 .

Step 3: Compute 
$y=j-d \times e(\bmod t)$

and the output $\operatorname{sign}_{\mathrm{d}}(\mathrm{m})=(\mathrm{e}, \mathrm{y})$.

Phase 4: Proxy multi-signature verification phase: When the verifier verifies the signature, he or she calculates the proxy public value Q corresponding to the proxy signature key $\mathrm{d}$ as

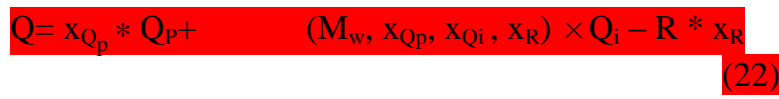

With the value, the verifier can confirm the validity of $\operatorname{Sig}_{d}(m)$ by validating the verification equality of the designated signature scheme.

\section{Step 1: Compute $\overline{\mathrm{J}}=\mathrm{y} \times \mathrm{B}+\mathrm{e} \times \mathrm{Q}=\left(\overline{\mathrm{J}}_{\mathrm{x}}, \overline{\mathrm{J}}_{\mathrm{y}}\right)$}

Step 2: And compute $\mathrm{e}^{\prime}=\mathrm{h}(\overline{\mathrm{J}} \mathrm{x}, \mathrm{m})$. Then check that $\mathrm{e}^{\prime}=$ $\mathrm{e}$ and if these are equal then valid signature otherwise not.

\section{CONCLUSIONS}

In this paper, we have reviewed improved $\mathrm{CCH} 1$ and improved $\mathrm{CCH} 2$ proxy multi-signature scheme based on elliptic curve cryptosystem. We have shown time complexity, space complexity and computational overhead of improved $\mathrm{CCH} 1$ and $\mathrm{CCH} 2$ proxy multisignature schemes. We have presented cryptanalysis of improved $\mathrm{CCH} 2$ proxy multi-signature scheme and showed that improved $\mathrm{CCH} 2$ scheme is suffered from various attacks i.e. the malicious original signers can forge the proxy signer's signature, forge a proxy signature and frame anyone who was never designated as proxy signer. In addition, we have proposed an enhanced proxy multi-signature scheme based on ECC. Enhanced scheme does not suffer from these given attacks.

\section{REFERENCES}

[1] V.S. Miller, "Uses of Elliptic Curves in Cryptography", Advances in Cryptology - Crypo'85, LNCS 218, pp. 417-426, 1985.

[2] N. Koblitz, "Elliptic Curve Cryptosystems", Mathematics of Computation (AMC), Vo1.48, No.177, pp. 203-209, 1987.

[3] M. Mambo, K. Usuda, and E. Okamoto, "Proxy Signatures for Delegating Signing Operation", Proc. 3rd ACM Conference on Computer and Communications Security, ACM press, pp.48-57, 1996.

[4] M. Mambo, K. Usuda, and E. Okamoto, "Proxy signature: Delegation of the power to sign messages", IEICE Trans. Fundamentals, Vol. E79A, No. 9, pp. 1338-1353, Sep. 1996.

[5] S. Kim, S. Park, and D. Won, "Proxy signatures, revisited", Information and Communications Security -ICICS'97, LNCS 1334, pp. 223-232, 1997.
[6] L. Yi, G. Bai and G. Xiao, "Proxy Multi-signature Scheme: A New Type of Proxy Signature", Electronics Letters, Vol. 36, Issue.6, pp. 527-528, 2000.

[7] H. M. Sun, "On Proxy Multisignature Schemes", Proceedings of the International Computer Symposium, pp.65-72, 2000.

[8] B. Lee, H. Kim, and K. Kim, "Strong proxy signature and its application", In Proceedings of SCIS, pp. 603- 608, 2001.

[9] T. S. Chen, T. P Liu, and Y. F Chung, "A ProxyProtected Proxy Signature Scheme Based on Elliptic Curve Cryptosystem", Proc. Of IEEE TENCOM, pp. 184-187, 2002.

[10] T.S. Chen, Y.F. Chung and G.S. Huang, "Efficient proxy multisignature schemes based on the elliptic curve cryptosystem", Computers \& Security, Elsevier, Vol. 22, No. 6, pp 527-534, 2003.

[11] T.S. Chen, Y.F. Chung, G.S. Huang, "A traceable proxy multi-signature scheme based on the elliptic curve cryptosystem", Applied Mathematics Computation, Elsevier, Vol. 1591 No. 1, pp 137 145, 2004.

[12]M.S. Hwang, S.F. Tzeng and C.S. Tsai, "Generalization of proxy signature based on elliptic curves", Computers Standard \& Interfaces, Elsevier, Vol. 26, Issue 2, pp. 73-84, 2004.

[13] G. L. Wang, F. Bao, J. Y. Zhou, and R. H. Deng, "Security Analysis of Some Proxy Signatures", Information Security and Cryptology, LNCS 2971, pp.305-319, 2004.

[14] S. Wang, G. Wang, F. Bao and J. Wang, "Cryptanalysis of A Proxy-Protected Proxy Signature scheme based on Elliptic Curve Cryptosystem”, IEEE, pp 3240-3243, 2004.

[15] J.H. Park, B.G. Kang, S. Park, "Cryptanalysis of Some Group-Oriented Proxy Signature Schemes", LNCS, vol. 3786, pp. 10-24, 2006.

[16]Feng Cao, Zhenfu Cao, "Cryptanalysis on a Proxy Multi-Signature Scheme", Proceeding of the first international Multi-symposiums on computer and computational Sciences(IMSCCS), IEEE, Vol. 2, pp. 117-120, 2006.

[17] M.H. Chang, I.T. Chen and M.T. Chen, "Design of Proxy Signature in ECDSA”, IEEE, 2008, pp 17-22, 2006.

[18] Fengying Li, Qingshui Xue, "Two Improved Proxy Multi-signature Schemes Based on the Elliptic Curve Cryptosystem", Communications in Computer and Information Science, Vol. 234, pp. 101-109, 2011.

[19] Sheetal Kalra, Sandeep K. Sood, "Elliptic Curve Cryptography: Survey and its Security

[20] Applications", ACAI Proceedings of the International Conference on Advances in Computing and Artificial Intelligence, ACM, pp 102-106,2011.

[21] I. Tutanescu, C. Anton, L. Ionescu and D. Caragata, "Elliptic Curves Cryptosystems Approaches", IEEE, pp 357-362, 2012. 
[22] Elliptic curve cryptography tutorial: http://www.tataelxsi.com/whitepapers/ECC_Tut_v1 _o.pdf

[23] Harsh Kumar Verma, Kamalpreet Kaur and Raman Kumar, "A Comparison of Threshold Proxy Signature Scheme", The 2008 International Conference on Security and Management, USA, 1417 July, 2008.

[24] Raman Kumar and Harsh Kumar Verma, "An Advanced Secure (t, n) Threshold Proxy Signature Scheme Based on RSA Cryptosystem for Known Signers", IEEE 2nd International Advance Computing Conference, Thapar University, India, 19-20 February, 2010.

[25] Raman Kumar and Harsh Kumar Verma, "Secure Threshold Proxy Signature Scheme Based on RSA for Known Signers", Journal of Information Assurance and Security, JIAS, Vol. 5, Issue 1, pp. 319-326, 2010.
Author(s) Biographies

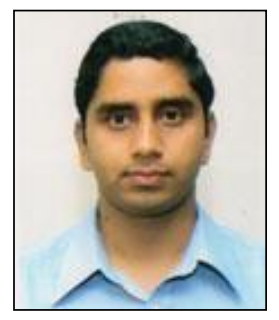

Mr. Raman Kumar (er.ramankumar@aol.in) is working as an Assistant Professor with the Department of Computer Science and Engineering, DAV Institute of Engineering and Technology, Jalandhar.

Before joining DAV Institute of Engineering and Technology, Jalandhar, He did his Bachelor of Technology with honours in Computer Science and Engineering from Guru Nanak Dev University; Amritsar (A 5 Star NAAC University). He did his Master of Technology with honours Computer Science and Engineering from Guru Nanak Dev University; Amritsar (A 5 Star NAAC University). His major area of research is Cryptography, Security Engineering and Information security. He has published many papers in refereed journals and conference proceedings on his research areas.

How to cite this paper: Raman Kumar,"Security Analysis and Implementation of an Improved Cch2 Proxy MultiSignature Scheme", IJCNIS, vol.6, no.4, pp.46-54, 2014. DOI: 10.5815/ijcnis.2014.04.06 